\title{
Measurement of the top quark mass at ATLAS
}

\author{
Diane Cinca*t \\ CNRS/IN2P3 - Laboratoire de Physique Corpusculaire (LPC) \\ Université Blaise Pascal \\ 24, avenue des Landais \\ BP 80026, 63171 Aubière Cedex - FRANCE \\ E-mail: diane.cinca@cern.ch
}

The top quark has been discovered in 1995 at Fermilab. Being the heaviest known elementary particle, it plays a special role in the Standard Model. The LHC produced first pp collisions at $7 \mathrm{TeV}$ centre-of-mass energies in fall 2009. The top quark was observed in both experiments ATLAS and CMS during 2010. First top mass measurement by ATLAS have been made using $35 \mathrm{pb}^{-1}$ of data recorded in 2010. Three separate direct measurements were performed in the semileptonic decay channel. All three compare data to templates from simulation at different mass points. The default method uses a stabilized top mass and the $R_{32}$ variable which is the ratio between reconstructed top mass and reconstructed $\mathrm{W}$ mass. The top mass measurement obtained for 2010 data is $m_{\text {top }}=(169.3+4.0+4.9) \mathrm{GeV} / \mathrm{c}^{2}$. The most important systematic uncertainty came from Jet Energy Scale the top mass being the invariant mass of three reconstructed jets. The luminosity increase will allow to record more that $1 \mathrm{fb}^{-1}$ by the end of the year 2011 .

The 2011 Europhysics Conference on High Energy Physics-HEP 2011,

July 21-27, 2011

Grenoble, Rhône-Alpes France

\footnotetext{
*Speaker.

†n behalf of the ATLAS Collaboration.
} 


\section{Introduction}

Two methods are presented to extract the top mass measurement. The top quark can be reconstructed from its decay products and its invariant mass extracted. But such a direct measurement is dependent on the simulation the top quark mass being dependent on the renormalisation scheme for QCD beyond leading-order predictions. Direct measurement will be presented in the second part while the first part presents a top mass extraction through cross section measurement.

\section{Top mass measurement from cross section}

By comparing the top pair production rate to the Standard Model theory, one can extract the top quark pole mass. In this analysis [1], theoretical cross section predictions are obtained from NNLO and NLO+NNLL approximations. The cross section is measured experimentally by using a topological discriminant method. The top quark mass is then extracted maximising a likelihood function composed of theoretical and experimental PDFs.

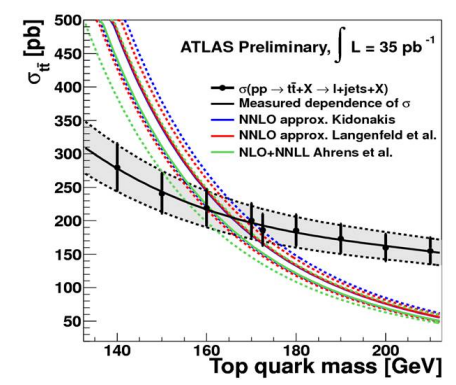

(a)

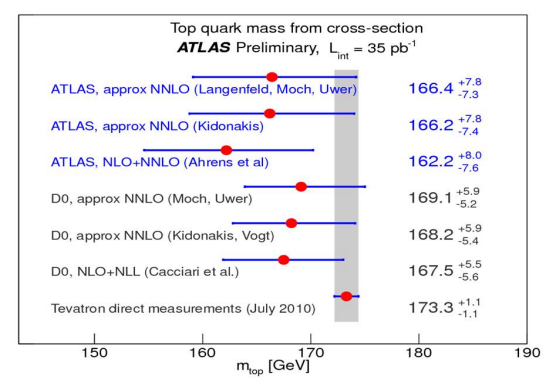

(b)

Figure 1: Left: Comparison between the expected evolution of $t \bar{t}$ cross section (blue, red and green) with respect to top pole mass hypothesis and the one observed on data (black). Right: Top pole mass extraction for different theoretical models compared to D0 experiment results.

\section{Semileptonic top event reconstruction}

Direct top mass measurement is dependent on the simulation. It needs to select the top decay products from the objects reconstructed in one event. The object selection used in these analyses is based on the semileptonic top pair signature. We require to have at least 4 jets reconstructed with at least on being b-tagged. We require exactly one isolated lepton (electron or muon) with a high transverse momentum. A significative missing transverse energy, correlated to the presence of a neutrino, must have been reconstructed. The hadronic top quark will be reconstructed by selecting the 3 jets which reconstruct the highest transverse momentum object.

\section{Direct top mass measurement}

The hadronic top quark decaying in 3 jets, it will be very sensitive to the jet energy scale. Three methods have been developed to reduce this sensitivity [2]. 


\subsection{1-D $R_{32}$ template method}

The main analysis reduces the sensitivity to the jet energy scale by measuring the ratio between the reconstructed hadronic top mass and the reconstructed hadronic $\mathrm{W}$ mass. This ratio is called $R_{32}=\frac{m_{t o p}^{r e c o}}{m_{W}^{\text {reco }}} . R_{32}$ references are developed for different top mass hypotheses and a likelihood maximisation is performed between data and the references to extract top mass measurement. The measurement obtained is $m_{t o p}=169.3 \pm 4.0($ stat $) \pm 4.9(\mathrm{syst}) \mathrm{GeV} / \mathrm{c}^{2}$, electron and muon channels added.

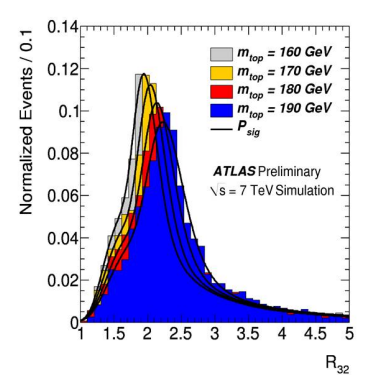

(a)

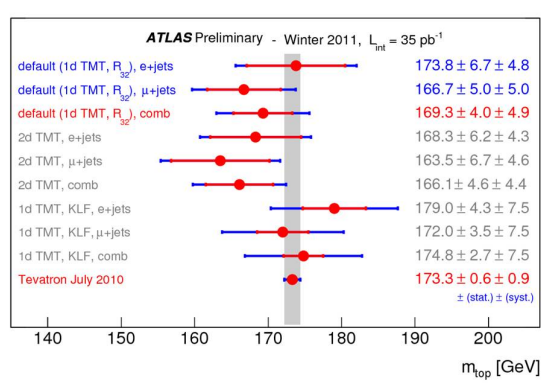

(b)

Figure 2: $R_{32}$ distribution for different top mass (left) hypotheses and top mass measurement from the three methods presented and compared to the reference of Tevatron result (right).

\subsection{2-D template method}

The 2-D template method uses the reconstructed hadronic $\mathrm{W}$ and top masses in a simultaneous measurement of the top mass and a global Jet Energy Scale factor (JSF). The hadronic W reconstruction is enhanced using a kinematical fit. The top mass and JSF are extracted using a template method and a likelihood maximisation between data and simulation. The top mass measurement obtained is $m_{t o p}=166.1 \pm 4.6($ stat $) \pm 4.4($ syst $) \mathrm{GeV} / \mathrm{c}^{2}$, electron and muon channels added.

\subsection{1-D kinematical fit analysis}

The third method uses a full kinematical fit of the semileptonic decay products. The reconstruction of the top pair is enhanced the kinematical fit using transfer functions to map the reconstructed objects to the parent ones and using constraints on both hadronic et leptonic top quarks. This method allows to obtain a very good resolution on the top mass but is sensitive to the jet energy scale. A template method and a likelihood maximisation are then used to extract the top mass measurement $m_{\text {top }}=173.8 \pm 2.7$ (stat) \pm 7.5 (syst $) \mathrm{GeV} / \mathrm{c}^{2}$. Figure 2 (right) shows the different top mass measurements obtained by the 3 methods presented in this proceeding with $35 \mathrm{pb}^{-1}$ of data.

\section{References}

[1] ATLAS Collaboration, ATLAS-CONF-2011-054, Determination of the top quark mass from the $t \bar{t}$ cross section measurement in pp collisions at $\sqrt{=7} \mathrm{TeV}$ with the ATLAS detector.

[2] ATLAS Collaboration, ATLAS-CONF-2011-033, Measurement of the top quark mass using the template method in pp collisions at $\sqrt{\equiv 7} \mathrm{TeV}$ with the ATLAS detector. 